\title{
Scleroderma Lung Involvement, Autoantibodies, and Outcome Prediction: The Confounding Effect of Time
}

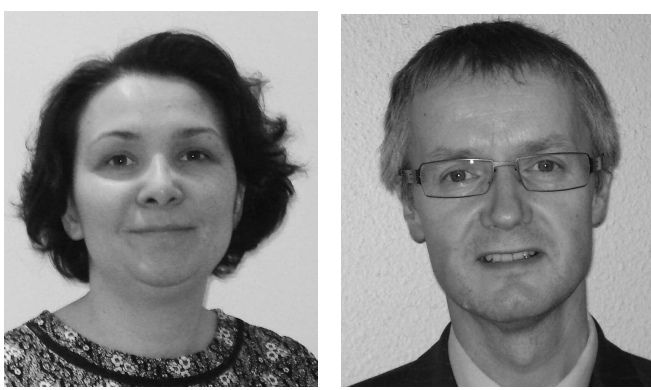

Systemic sclerosis $(\mathrm{SSc})$ remains a poorly understood disease and so far none of the routinely used immunosuppressive treatments has been definitively shown to benefit longterm disease outcome. Scleroderma-related cardiopulmonary involvement has been the leading cause of death in more recent decades, and pulmonary fibrosis $(\mathrm{PF})$ and pulmonary hypertension $(\mathrm{PH})$ account for a substantial proportion of the SSc-related deaths, as demonstrated by several large metaanalyses $1,2,3$.

Early detection and monitoring of $\mathrm{PF}$ and $\mathrm{PH}$ may benefit outcome by permitting earlier intervention in severe or progressive cases ${ }^{4}$. Multiple attempts have been made to develop prediction models, both for development and for outcome, in already present SSc-related lung disease. Consistently, autoantibody specificities are among the strongest but not the only predictors of organ disease in scleroderma patients, and autoantibody characterization is a mandatory part of investigations in new SSc cases.

It is well established that while positivity for anticentromere antibodies (ACA) is associated with the limited cutaneous subset of the disease and a low risk of pulmonary or renal involvement, presence of antitopoisomerase I antibodies (ATA) conveys a substantially increased risk of PF development; moreover anti-RNA polymerase antibodies (ARA) are strongly associated with the diffuse cutaneous subset (dcSSc) and development of scleroderma renal crisis (SRC) $)^{5}$. Most cohort studies describe no particular association between ARA positivity and PH or PF development. Although this was true in an unadjusted analysis, when correcting for other variables, ARA positivity was shown to associate with an increase in the hazard of PH development in a large single-center cohort analysis ${ }^{6}$. In addition, compared to ACA-positive patients, those carrying ARA have been shown to develop PF more frequently ${ }^{7,8}$.

Several studies have assessed and compared the value of pulmonary function tests (PFT) and high-resolution computed tomography (HRCT) for the screening and monitoring of PF, and even though HRCT appears to be a more accurate tool ${ }^{9,10}$, the associated cost and radiation burden mean that serial PFT remains the preferred method for longterm PF monitoring. An algorithm combining results from HRCT and PFT was developed by Goh, et al and seems to have a very good prognostic value, allowing for an easy and quick staging of lung disease and assessment of the need for immunosuppressive treatment ${ }^{11}$.

In this issue of The Journal, the article by Hoffman-Vold, et al focuses on SSc-related cardiopulmonary complications in a group of patients with well-characterized autoantibody profiles and looks for specific patterns associated with ARA positivity ${ }^{12}$. This is a prospective observational study, presenting a cohort of SSc patients with very robust associated clinical data. Lung complications have been ascertained using gold-standard tests with serial PFT and HRCT scanning used to define PF, while all PH cases were confirmed using right heart catheterization.

Similar to previous cohort studies, the authors find no association between ARA positivity and the overall risk of development of PF: nearly half of the ARA-positive patients (49\%) showed no evidence of PF development over the duration of the study. Nevertheless, $18 \%$ of the ARA-positive patients did develop extensive PF, affecting more than $20 \%$ of the lung on HRCT, emphasizing the importance of PF screening in all SSc patients, irrespective of their presumed risk of this complication.

Moreover, a particularly interesting finding is that among ARA-positive patients who did develop PF, extensive fibrosis tended to develop later on in the disease course. This was unlike the ATA-positive cohort, in which the great majority of subjects who were to develop extensive PF already had it at baseline. This is in keeping with the findings of a recent observational cohort study of 294 patients from Thailand, where disease duration at first detection of PF was much shorter in ATA-positive compared to ATA-negative patients $^{13}$. It also reflects the fact that association between

\section{See Cardiopulmonary disease in anti-RNAP+ SSc, page 459}


PF development and ATA positivity has a significant interaction with disease duration, with hazard of PF doubling after 5 years of disease ${ }^{6}$.

There could be multiple explanations for the apparently different course of progression in PF in subjects with ATA and ARA, and different pathophysiological mechanisms for fibrotic change development are likely. For ATA-positive patients these may be autoimmune inflammation-driven, while in patients with ARA, PF progression could be perpetuated by gastroesophageal reflux, with associated microaspiration or recurrent infections, leading to alveolar epithelial injury occurring later in the disease course, which could account for the later development of extensive disease in those subjects ${ }^{14,15}$.

One major confounder in the study by Hoffman-Vold, et $a l$ is the significant difference in disease duration at study baseline between the ARA-positive and ATA-positive patients, where those carrying ATA had on average 3 years longer disease duration than subjects carrying ARA. Given that clinically significant PF generally develops early in the disease - and multiple studies have shown that this happens to the majority of patients within the first 5 years from onset $^{6,16,17}$ - it is not surprising that most ATA-positive patients who were going to develop significant PF had already reached this endpoint, given that mean disease duration at baseline in this group was 6.2 years, compared to 3.3 years for ARA-positive patients. This highlights the importance of accounting for disease duration in any research into outcome prediction in patients with SSc.

SSc has a very specific natural pattern of progression. As a result, the timing of peak skin disease and major organ complications, if they are to develop, could be anticipated. Thus, skin involvement in patients with dcSSc, as measured by the modified Rodnan skin score (mRSS), tends to worsen comparatively rapidly in the initial stages of the disease. The majority of patients with the diffuse subset of SSc reach their peak mRSS within the first 3 years from first non-Raynaud symptom, and skin tends to improve thereafter in about $80 \%$ of the subjects ${ }^{18,19}$. Deterioration in skin disease or new development of worsening skin thickening in patients with disease duration longer than 5 years is extremely rare and often a sign of an underlying more sinister pathology.

Similar to the natural evolution of skin disease, major organ complications, with the obvious exception of $\mathrm{PH}$, normally develop in the early stages of SSc and could even be a presenting feature ${ }^{5,18}$. For example, incidence of SRC is highest in the first 3 years of disease, significantly lessening in later years, with only anecdotal cases of SRC developing 10 years or longer after SSc onset ${ }^{4}$. Clinically significant $\mathrm{PF}$ [defined as forced vital capacity (FVC) or $\mathrm{DLCO}<55 \%$ or a drop in FVC or DLCO $>15 \%$ from first assessment] also tends to develop earlier on, with over twothirds of the subjects who are to develop this endpoint reaching it in the first 5 years from disease onset ${ }^{6}$. On the other hand, PH does develop later in the disease course, with an apparently constant incidence rate over time, suggesting that longer followup will be associated with a greater probability of PH development in a given subject.

As a result, any study investigating predictors of organ complications in SSc should take into account the timing of those complications and the disease duration in the study subjects. Time-to-event analysis rather than simple associations between explanatory variables and present/absent outcomes should be considered, and interaction between any of the predictor variables and time should be tested as appropriate.

In this context, the study by Hoffman-Vold, et al is a good illustration of many of the challenges associated with studying a rare disease with variable presentation, and a specific course of progression that can affect the studied associations if not adjusted for in the analysis. In this case, although the numbers were reasonable for a scleroderma study, they were still insufficient to allow for a comprehensive multivariable analysis. One inevitable conclusion from this article is that there is a real need for even more detailed and larger studies, assessing the effect of autoantibody specificities on the outcome of lung complications in patients with SSc. At a practical level, these findings reassert the clinical importance of ongoing monitoring of patients with SSc for potential lung complication development.

SVETLANA I. NIHTYANOVA, MD, Clinical Research Fellow in Rheumatology; CHRISTOPHER P. DENTON, FRCP, Professor of Experimental Rheumatology and Consultant Rheumatologist, Centre for Rheumatology and Connective Tissue Diseases, UCL Medical School, Royal Free Campus, London, UK.

Address correspondence to Dr. S.I. Nihtyanova, Centre for Rheumatology and Connective Tissue Diseases, UCL Medical School, Royal Free Campus, Rowland Hill Street, London NW3 2PF, UK.

E-mail: s.nihtyanova@ucl.ac.uk

\section{REFERENCES}

1. Komócsi A, Vorobcsuk A, Faludi R, Pintér T, Lenkey Z, Költő G, et al. The impact of cardiopulmonary manifestations on the mortality of SSc: a systematic review and meta-analysis of observational studies. Rheumatology 2012;51:1027-36.

2. Elhai M, Meune C, Avouac J, Kahan A, Allanore Y. Trends in mortality in patients with systemic sclerosis over 40 years: a systematic review and meta-analysis of cohort studies. Rheumatology 2012;51:1017-26.

3. Rubio-Rivas M, Royo C, Simeon CP, Corbella X, Fonollosa V. Mortality and survival in systemic sclerosis: systematic review and meta-analysis. Semin Arthritis Rheum 2014;44:208-19.

4. Nihtyanova SI, Tang EC, Coghlan JG, Wells AU, Black CM, Denton CP. Improved survival in systemic sclerosis is associated with better ascertainment of internal organ disease: a retrospective cohort study. QJM 2010;103:109-15.

5. Nihtyanova SI, Denton CP. Autoantibodies as predictive tools in systemic sclerosis. Nature Rev Rheumatol 2010;6:112-6.

6. Nihtyanova SI, Schreiber BE, Ong VH, Rosenberg D, Moinzadeh P, Coghlan JG, et al. Prediction of pulmonary complications and

Personal non-commercial use only. The Journal of Rheumatology Copyright @ 2017 . All rights reserved. 
long-term survival in systemic sclerosis. Arthritis Rheumatol 2014;66:1625-35.

7. Motegi S, Toki S, Yamada K, Uchiyama A, Ishikawa O. Demographic and clinical features of systemic sclerosis patients with anti-RNA polymerase III antibodies. J Dermatol 2015; 42:189-92.

8. Airo P, Ceribelli A, Cavazzana I, Taraborelli M, Zingarelli S, Franceschini F. Malignancies in Italian patients with systemic sclerosis positive for anti-RNA polymerase III antibodies. J Rheumatol 2011;3:1329-34.

9. Wangkaew S, Euathrongchit J, Wattanawittawas P, Kasitanon N . Correlation of delta high-resolution computed tomography (HRCT) score with delta clinical variables in early systemic sclerosis (SSc) patients. Quant Imaging Med Surg 2016;6:381-90.

10. Suliman YA, Dobrota R, Huscher D, Nguyen-Kim TD, Maurer B, Jordan S, et al. Brief report: pulmonary function tests: high rate of false-negative results in the early detection and screening of scleroderma-related interstitial lung disease. Arthritis Rheumatol 2015;67:3256-61.

11. Goh NS, Desai SR, Veeraraghavan S, Hansell DM, Copley SJ, Maher TM, et al. Interstitial lung disease in systemic sclerosis: a simple staging system. Am J Respir Crit Care Med 2008; 177:1248-54

12. Hoffmann-Vold AM, Midtvedt $\varnothing$, Tennøe AH, Garen T, Lund MB, Aaløkken TM, et al. Cardiopulmonary disease development in anti-RNA polymerase III positive systemic sclerosis; comparative analyses from an unselected, prospective patient cohort. J Rheumatol 2017;44:459-65.
13. Foocharoen C, Suwannachat P, Netwijitpan S, Mahakkanukrauh A, Suwannaroj S, Nanagara R. Clinical differences between Thai systemic sclerosis patients with positive versus negative anti-topoisomerase I. Int J Rheum Dis 2016;19:312-20.

14. Christmann RB, Wells AU, Capelozzi VL, Silver RM. Gastroesophageal reflux incites interstitial lung disease in systemic sclerosis: clinical, radiologic, histopathologic, and treatment evidence. Semin Arthritis Rheum 2010;40:241-9.

15. Furnari M, Savarino V, de Bortoli N, Savarino E. Interstitial lung disease in systemic sclerosis patients may benefit more from anti-reflux therapies than from immunosuppressants. Autoimmun Rev 2016;15:1208-9.

16. Steen VD, Conte C, Owens GR, Medsger TA Jr. Severe restrictive lung disease in systemic sclerosis. Arthritis Rheum 1994;37:1283-9.

17. Steen VD, Medsger TA Jr. Severe organ involvement in systemic sclerosis with diffuse scleroderma. Arthritis Rheum 2000; 43:2437-44.

18. Medsger TA Jr. Natural history of systemic sclerosis and the assessment of disease activity, severity, functional status, and psychologic well-being. Rheum Dis Clin N Am 2003;29:255-73, vi.

19. Shand L, Lunt M, Nihtyanova S, Hoseini M, Silman A, Black CM, et al. Relationship between change in skin score and disease outcome in diffuse cutaneous systemic sclerosis: application of a latent linear trajectory model. Arthritis Rheum 2007;56:2422-31.

J Rheumatol 2017;44:404-6; doi:10.3899/jrheum.170055 